\title{
Nachhaltigkeitsbericht mit Bürgerbeteiligung
}

Die Nachhaltigkeit einer kommunalen Entwicklung kann durch Indikatoren abgebildet werden. In Pleidelsheim geschieht dies im Rahmen des Prozesses der Lokalen Agenda 21, in dem Bürger und die Verwaltung zusammenarbeiten. In den Jahren 2003 und 2008 wurden dabei umfangreiche Nachhaltigkeitsberichte erstellt. Von Annegret Bartenbach und Bernhard Thinschmidt

D ie Gemeinde Pleidelsheim ist 1997 in den Prozess der Lokalen Agenda 21 (LA21) eingetreten. Der Prozess wird gemeinsam von der Verwaltung und den Bürger(inne)n gestaltet und vom Gemeinderat unterstützt. Auf der Basis der Nachhaltigkeitsstrategie der Bundesregierung wurde 2001 das Leitbild für eine nachhaltige Entwicklung für Pleidelsheim mit den Schwerpunkten Generationengerechtigkeit, Lebensqualität und sozialer Zusammenhalt entwickelt und am 15. Mai 2003 vom Gemeinderat verabschiedet.

\section{Auf dem Weg zum kommunalen Nachhaltigkeitsbericht ...}

Bereits während der Leitbilderstellung wurde klar, dass die unterschiedlichen Ziele regelmäßig überprüft und Fortschritte beschrieben werden müssen. Die guten Erfahrungen mit der Bürgerbeteiligung bei der Leitbilderstellung, die geringen zur Verfügung stehenden finanziellen und personellen Mittel sowie die hohe Motivation der Aktiven in der LA21 waren ausschlaggebend dafür, das Projekt „Nachhaltigkeitsbericht für Pleidelsheim“ ebenfalls ehrenamtlich durchzuführen.

Wie beim Leitbild sollten möglichst viele Bürger(innen) beteiligt werden. Ebenso hatte sich gezeigt, dass ein so komplexes Thema zunächst in kleinere Portionen gegliedert und auf konkrete Fragestellungen heruntergebrochen werden muss, bevor selbst motivierte Laien aktiv mitarbeiten und eine Diskussion in breiterem Rahmen mit produktivem Ergebnis stattfinden kann. Daher wurde von einer Kerngruppe nach intensiver Auseinandersetzung mit dem Thema Nachhaltigkeitsbericht ein Vorschlag erarbeitet. Als Basis diente ein allgemeiner Indikatoren-Leitfaden, der eine komplette Vorlage inklusive Definitionen und Layout bietet (Umweltministerien 2001).

Die im Leitfaden vorgeschlagenen Indikatoren wurden an den Leitbildzielen der Gemeinde Pleidelsheim gespiegelt.
Viele Indikatoren konnten direkt übernommen werden. Andere wurden angepasst oder erweitert. Für einige Ziele mussten zusätzliche Indikatoren gefunden werden, sodass schließlich eine umfangreiche Liste vorlag. Davon mussten wegen fehlender Datengrundlagen oder aufwendiger Ermittlung verschiedene Indikatoren entfallen. Die wichtigsten Indikatoren wurden dann einzelnen Autor(inn)en zur Bearbeitung übergeben.

Die Autor(inn)en ermittelten die entsprechenden Daten, passten die im Leitfaden beschriebene Zielsetzung an das Leitbild an und interpretierten die Entwicklung des Indikators. Die Indikatorenentwürfe wurden anschließend durch Lektor(inn)en inhaltlich kommentiert und in der LA21-Gruppe überarbeitet und beschlossen.

\section{... müssen alle Akteure an einem Strang ziehen}

Basis des Leitbild- und Nachhaltigkeitsberichts war das gemeinsame Vorgehen der drei Akteursgruppen Bürger(innen), Bürgermeister und Verwaltung innerhalb des LA21-Prozesses. Ohne dieses Miteinander wäre der Bericht nicht zustande gekommen. Das ehrenamtliche Engagement Einzelner trug wesentlich zum Gelingen bei. Selbst nach dem zweiten Bericht zeigt sich, dass Nachhaltigkeit ein abstrakter Begriff bleibt und nur wenige Bürger(innen) damit erreicht werden.

Ein Interesse den Nachhaltigkeitsbericht regelmäßig fortzuschreiben haben in erster Linie Entscheidungsträger der Kommune und Bürger(innen), denen die Entwicklung im Sinne der LA21 wichtig ist. Wir in der Gemeinde Pleidelsheim sind fest davon überzeugt, dass dies ein wichtiges Instrument auf dem Weg in eine generationengerechte, nachhaltige Zukunft ist.

\section{Literatur}

Umweltministerien der Länder Baden-Württemberg, Bayern, Hessen und Thüringen (Hrsg.): „Leitfaden: Indikatoren im Rahmen einer Lokalen Agenda 21 “, 2001.

\section{AUTOREN + KONTAKT}

Bernhard Thinschmidt ist einer der ehrenamtlich Engagierten innerhalb der Lokalen Agenda 21 Pleidelsheim; Annegret Bartenbach ist Umweltamtsleiterin der Gemeinde Pleidelsheim und zuständig für den Prozess der Lokalen Agenda 21.

Gemeinde Pleidelsheim, Marbacher Str. 5, 74385 Pleidelsheim. Tel.: +49 7144 26427; E-Mail: a.bartenbach@rathaus-pleidelsheim.de
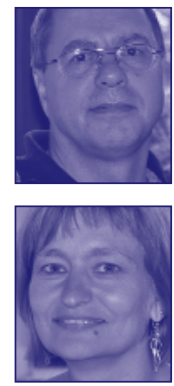
(c) 20I0 Authors; licensee IÖW and oekom verlag. This is an article distributed under the terms of the Creative Commons Attribution Non-Commercial No Derivates License (http://creativecommons.org/licenses/by-nc-nd/3.o/), which permits unrestricted use, distribution, and reproduction in any medium, provided the original work is properly cited. 